\title{
EDITORIAL
}

\section{Hacia las III Jornadas de Discusión sobre las Revistas Científicas Arbitradas}

El2002 es el séptimo año de edición ininterrumpida y progresiva de la Revista Venezolana de Gerencia. El incremento de tres a cuatro números al año, requirió la ampliación del equipo de trabajo. Por lo tanto, comenzamos este año con la incorporación al Comité Editorial de las investigadoras: Mirtha López Valladares, Lilia Pereira de Homes y Nelly Primera, todas acreditadas en el Programa de Promoción al Investigador (PPI) del FONDO NACIONAL DE INVESTIGACIONES CIENTÍFICAS Y TECNOLÓGICAS (FONACIT), somos así seis miembros del Comité Editorial.

Se inicia el año con el número 17. Ocho artículos evidencian el gran pluralismo ideológico que caracteriza a la revista; consideramos que no puede ser de otra manera en tanto es un requisito para el avance científico en un tema como la Gerencia, invadido por un solo pensamiento.

El primer trabajo es del investigador cubano Mario González Arencibia titulado "Modificaciones en la relación trabajo-capital: Sus efectos en el proceso de producción de plusvalía en la era de la Globalización"; el trabajo pone sobre el tapete que en el contexto de la globalización y al rebasar lo nacional el proceso de conformación del proletariado industrial, es necesario replantear el cuerpo teórico de la economía política marxista en lo referente a la acumulación de capital en el espacio de lo nacional.

Se incluyen dos artículos sobre gerencia educativa respecto a la realidad venezolana, ambos de gran pertinencia cuando en el país se intensifican la presiones por la reforma de la educación superior. El trabajo del Dr. Luis Fuenmayor Toro, ex-Rector de la Universidad Central de Venezuela y actual director de la Oficina de Planificación del Sector Universitario, titulado "A propósito de las iniquidades en el ingreso a la educación superior en Venezuela", expone los resultados de una investigación donde estudia la injusticia en la admisión de estudiantes a las universidades oficiales del país y cuestiona el rol de éstas en dicho problema. Además, la Dra. Alicia Inciarte, quien además de investigadora tiene una amplia y exitosa experiencia como directora de postgrado con nivel doctoral en la Universidad del Zulia, pone a discusión de la comunidad científica el trabajo titulado "Gerenciar el cambio en la formación de Postgrado en Venezuela", el cual realiza una crítica constructiva de la orientación y práctica de la producción de conocimiento en los post-grados de nuestro país. 
Otros trabajos que dan cuenta del avance científico gerencial en Venezuela son, por una parte, el trabajo titulado "Agroforestería y Etica Ambiental en la Gerencia de Sistemas de Producción", de los investigadores Juan José Pérez e Isneira Huerta, ambos de la Facultad de Agronomía de La Universidad del Zulia, quienes desde una perspectiva de búsqueda de equilibrio ecológico discuten sobre la ética ambiental y la agroforestería en los sistemas de producción agrícola. Por otra parte, la Dra. Margalit Berlin, investigadora de la Universidad Simón Bolívar presenta los resultados de una investigación sobre la cultura organizacional, tema de gran relevancia en este momento; aborda concretamente el choque entre culturas gerenciales en una joint venture venezolana. Por último, el trabajo titulado Gestión de Calidad y su dimensión ético-competitiva en la Pequeña y Mediana Empresa Metalmecánica de la Región Zuliana de los investigadores Juliana Ferrer, José Luis Vázquez, Caterina Clemenza y Rosario Pérez, exponen en el cual estudian las limitaciones de las mencionadas empresas para garantizar su perpetuidad.

Finalmente están dos trabajos de investigadoras argentinas, uno, titulado "Persistencias e Incentivos en la Argentina de los 90: Los cambios institucionales en las políticas sociales", de Karina Ansolabehere, investigadora del Instituto Gino Germani, quien realiza actualmente su doctorado en FLACSO-México; desde una perspectiva neoinstitucional, explora las heterogeneidades en la descentralización de las políticas sociales en Argentina. Por su parte, Josefina Vaca, investigadora de la Universidad de Quilmes, en el trabajo titulado "Las Tecnologías de la Información y la Comunicación en Argentina. Un enfoque regional", busca respuesta a ¿qué características asume el proceso de incorporación de las tecnologías de la Información y Comunicación (TIC's) en Argentina?

Cuando ponemos a disposición de la comunidad científica estos aportes latinoamericanos, nos preparamos a participar activamente en las TERCERAS JORNADAS DE DISCU. SIÓN SOBRE LA GESTION DE LAS REVISTAS CIENTÍFICAS ARBITRADAS. Este evento comenzó a realizarse en el año 2000 por iniciativa de la revista Espacio Abierto de la Universidad del Zulia, fue asumido en el 2001 por la Revista de Ciencias Sociales, también de la Universidad del Zulia y corresponde este año a la Revista Ciencias de Gobierno, del Instituto Zuliano de Estudios Políticos, Económicos y Sociales (IZEPES).

Al igual que en los años anteriores, el evento tiene como propósito discutir sobre los principales problemas con los cuales se enfrenta la edición y distribución de revistas científicas. Temas como: indización, arbitraje, calidad, pertinencia científica, costos y medios de difusión entre otros, serán puestos a discusión por conferencistas centrales y ponentes en mesas de trabajo. Invitamos a los editores a incorporarse activamente en este evento, el cual siempre ha contado con participación de varios países de América Latina. Las Jornadas serán realizadas en el segundo semestre del presente año, mayor información puede ser obtenida a través del siguiente E-mail: izepes@iamnet.com 